\title{
Mechanism of NKT cell activation by intranasal coadministration of $\alpha$-galactosylceramide, which can induce cross-protection against influenza viruses
}

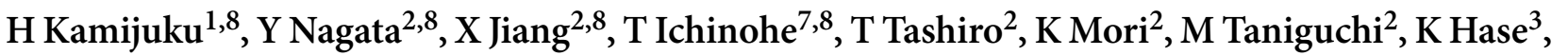

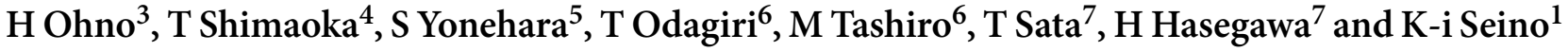

In a nasal vaccine against influenza, the activation of natural killer T (NKT) cells by intranasal coadministration of $\alpha$-galactosylceramide ( $\alpha$-GalCer) can potently enhance protective immune responses. The results of this study show that the NKT cell-activated nasal vaccine can induce an effective cross-protection against different strains of influenza virus, including $\mathrm{H} 5$ type. To analyze the mechanism of NKT cell activation by this nasal vaccine, we prepared fluorescencelabeled $\alpha$-GalCer by which we detect a direct interaction between NKT cells and $\alpha$-GalCer-stored dendritic cells in nasal mucosa-associated tissues. Accordingly, although very few NKT cells exist at mucosa, the nasal vaccination induced a localized increase in NKT cell population, which is partly dependent on CXCL16/CXCR6. Furthermore, we found that NKT cell activation stimulates mucosal IgA production by a mechanism that is dependent on interleukin (IL)-4 production. These results strengthen the basis of nasal vaccination via NKT cell activation, which can induce immune cross-protection.

\section{INTRODUCTION}

Influenza is a highly contagious and serious viral infection of the respiratory tract that can lead to annual epidemics of influenza. Lethal pneumonia and encephalopathy caused by influenza virus are becoming critical health hazards, particularly among children and elderly persons. ${ }^{1}$ Furthermore, the emergence of $\mathrm{H} 5 \mathrm{~N} 1$ influenza in bird populations is believed to be a potential of new source of pandemic influenza infections in humans. ${ }^{2}$ Influenza vaccines generally comprise a hemagglutinin (HA) antigen fraction of the inactivated viruses (inactivated split vaccines) and are administered parenterally to induce serum anti-HA immunoglobulin G (IgG). Such vaccines are highly protective against homologous virus infection but generally less effective against heterologous virus infection. ${ }^{3}$ In contrast, numerous precedent studies have shown that mucosal immunity acquired either via natural infection or via nasal vaccination with weakened viral agents is more effective and cross-protective against subsequent viral infection than the systemic immunity induced by parenteral vaccines in humans and mice. ${ }^{4,5}$ Cross-protection is one of the key advantages of mucosal immunity and is thought to be mediated by a complex mechanism comprising both cellular and humoral immunity. ${ }^{6}$ However, recent studies by several research groups have highlighted the importance of antigen-specific antibodies, particularly secretory IgA in the respiratory tract in the case of influenza virus infection. ${ }^{7-12}$ Accordingly, the induction of a high titer of the secreted form of IgA in the respiratory tract is advantageous for protection against unpredictable epidemics of influenza, including H5N1. One way to accomplish this is via intranasal (IN) vaccination whereby secretion of effective levels of specific IgA is induced in the nasal mucosa and the respiratory tract.

\footnotetext{
${ }^{1}$ Division of Bioregulation Research, Institute of Medical Science, St Marianna University School of Medicine, Miyamae-ku, Kawasaki City, Kanagawa, Japan. ${ }^{2}$ Laboratory for Immune Regulation, RIKEN Research Center for Allergy and Immunology, Tsurumi-ku, Yokohama City, Kanagawa, Japan. ${ }^{3}$ Laboratory for Epithelial Immunobiology, RIKEN Research Center for Allergy and Immunology, Tsurumi-ku, Yokohama City, Kanagawa, Japan. ${ }^{4}$ Molecular Preventive Medicine, Graduate School of Medicine, University of Tokyo, Bunkyo-ku, Tokyo, Japan. ${ }^{5}$ Graduate School of Biostudies, Kyoto University, Sakyo-ku, Kyoto, Japan. ${ }^{6}$ Department of Virology III, National Institute of Infectious Diseases, Musashimurayama City, Tokyo, Japan. ${ }^{7}$ Department of Pathology, National Institute of Infectious Diseases, Musashimurayama City, Tokyo, Japan. 8These authors contributed equally to this work. Correspondence: K-i Seino (seino@marianna-u.ac.jp) or H Hasegawa (hasegawa@nih.go.jp) 
Nasal

infection
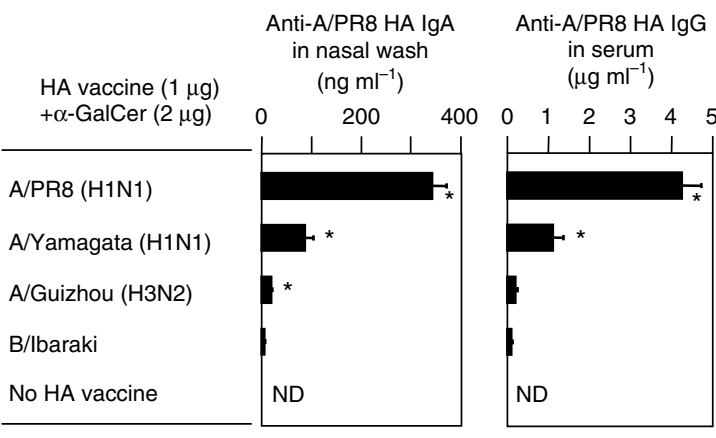

Anti-A/PR8 HA IgG Serum HI titers to $A / P R 8$ virus

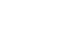

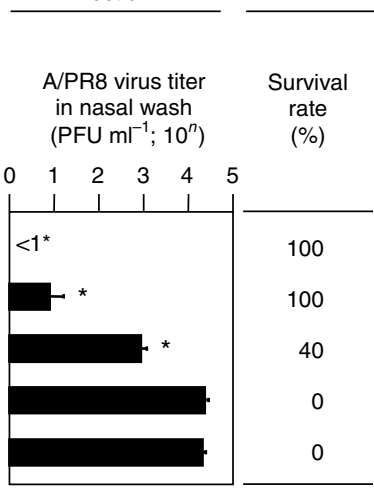

Lethal pneumonia

b Intramuscular

\begin{tabular}{l|l|}
\multicolumn{1}{c|}{$\begin{array}{c}\text { Anti-A/PR8 HA IgA } \\
\text { in nasal wash } \\
\text { HA vaccine }(1 \mu \mathrm{g}) \\
+\alpha \text {-GalCer }(2 \mu \mathrm{g})\end{array}$} & $\begin{array}{l}\left(\mathrm{ng} \mathrm{m}^{-1}\right) \\
\text { A/PR8 (H1N1) }\end{array}$ \\
\hline A/Yamagata (H1N1) & ND \\
A/Guizhou (H3N2) & ND \\
B/lbaraki & ND \\
No HA vaccine & ND \\
\hline
\end{tabular}

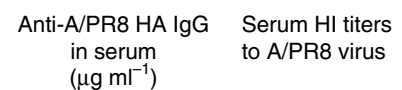

Anti-A/PR8 HA IgG Serum HI titers in serum to $A / P R 8$ virus $\left(\mu \mathrm{g} \mathrm{ml}^{-1}\right)$
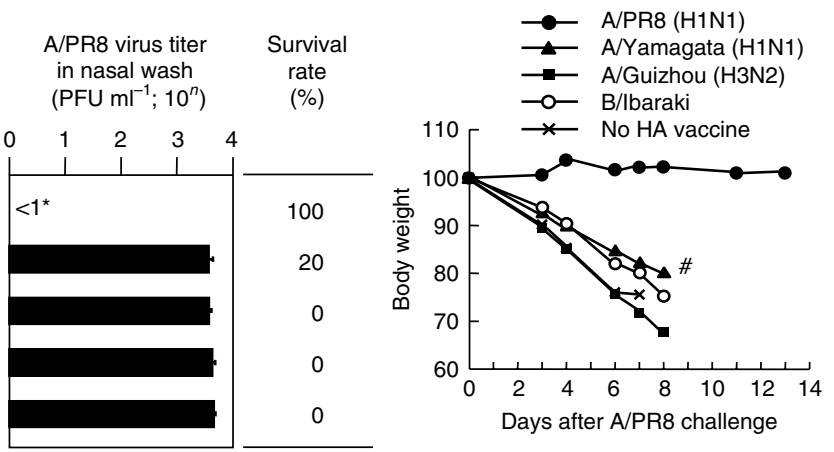

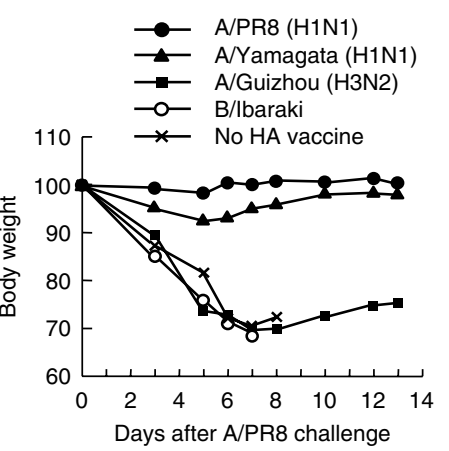

c

Immunization (IN)

\begin{tabular}{l} 
HA vaccine $(1 \mu \mathrm{g})$ \\
$+\alpha-$ GalCer$(2 \mu \mathrm{g})$ \\
\hline A/PR8 (H1N1) \\
No HA vaccine \\
A/PR8 (H1N1) \\
No HA vaccine \\
A/PR8 (H1N1) \\
No HA vaccine \\
A/PR8 (H1N1) \\
No HA vaccine
\end{tabular}

\begin{tabular}{lr}
\multicolumn{2}{c}{$\begin{array}{c}\text { Serum HI titers to the } \\
\text { indicated viruses }\end{array}$} \\
\hline A/PR8 (H1N1) & 1,280 \\
A/PR8 (H1N1) & $<10$ \\
A/Yamagata (H1N1) & 320 \\
A/Yamagata (H1N1) & $<10$ \\
A/Guizhou (H3N2) & $<10$ \\
A/Guizhou (H3N2) & $<10$ \\
B/lbaraki & $<10$ \\
B/lbaraki & $<10$
\end{tabular}

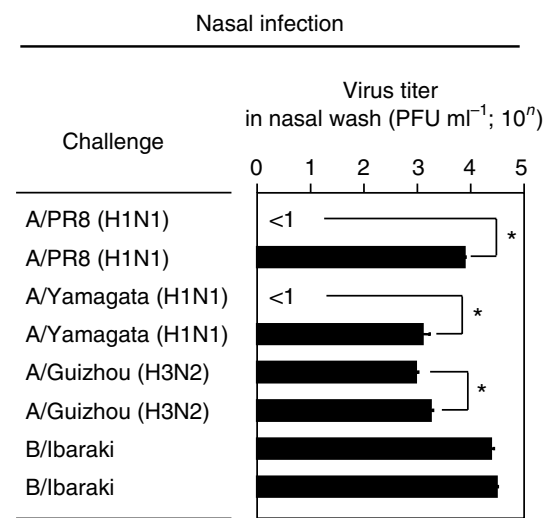

Figure 1 Cross-protective antibody responses against A/PR8 HA. Five mice per group were IN (a) or IM (b) immunized with A/PR8 (H1N1), A/Yamagata (H1N1), A/Guizhou (H3N2), or B/lbaraki HA vaccine with $\alpha$-GalCer as an adjuvant twice with a 4-week interval. The nasal wash and serum samples were collected 2 weeks after the second immunization, and anti-A/PR8 HA-specific IgA and IgG titers and serum HI titers to A/PR8 virus were measured. Another set of mice was IN infected with 1,000 PFU of A/PR8 virus in $1 \mu$ I PBS 2 weeks after the second immunization. The nasal wash fluid was collected 3 days after virus challenge. The virus titer was measured by plaque assay. In the influenza virus-induced lethal pneumonia model, the immunized mice were challenged with a lethal dose $\left(1,000 \mathrm{PFU}\right.$ in $\left.20 \mu \mathrm{l}: 40 \mathrm{LD}_{50}\right)$ of A/PR8 virus at 2 weeks after the second immunization. The body weight and survival rate of the mice were then estimated daily. The survival rate at day 14 is indicated. For the body-weight change, each point represents the relative ratio of initial body weight (mean) of five mice for each day after the challenge. One mouse (no. $1 ; 20 \%)$ recovered thereafter. (c) Five mice per group were IN immunized twice with A/PR8 (H1N1) plus $\alpha$-GalCer. Serum HI titers to the indicated viruses and nasal virus titers after challenge of the indicated viruses are shown. "No HA vaccine" group received only $\alpha$-GalCer. All the experiments were repeated independently at least three times, with similar results. Each column represents the mean \pm s.d. ${ }^{*} P<0.01$ vs. group of no HA vaccine. HA, hemagglutinin; $\mathrm{HI}$, hemagglutination inhibition; IN, intranasal; ND, not detected; PBS, phosphate-buffered saline.

In developing IN vaccines, cholera toxin (CT) and Escherichia coli heat-labile toxin have been proposed as adjuvants to enhance mucosal immune responses. ${ }^{13}$ Although CT and $E$. coli heat-labile toxin are effective adjuvants to induce mucosal immune responses, they also cause adverse events in humans, including nasal discharge $\mathrm{e}^{14}$ and facial nerve palsy. ${ }^{15}$ Therefore, a clinical need exists for the development of novel adjuvants for IN vaccines that not only are as effective as CT or E. coli heat-labile toxin but also are acceptably safe for human use. 
Natural killer T (NKT) cells are a specific subset of immune regulatory cells that express an invariant antigen receptor $\alpha$-chain encoded by a V $\alpha 14-J \alpha 281$ rearranged gene segment in mice and V $\alpha 24-\mathrm{J} \alpha 18$ in humans. ${ }^{16}$ NKT cells can be directly activated by glycolipids such as $\alpha$-galactosylceramide ( $\alpha$-GalCer) presented by CD1d. ${ }^{17}$ Upon activation, NKT cells rapidly produce both Th1- and Th2-type cytokines, including interferon- $\gamma$ (IFN- $\gamma$ ) and interleukin (IL)-4, and can contribute to the upregulation of both cellular and humoral immune responses. ${ }^{18}$ It was recently reported that $\alpha$-GalCer can serve as an effective adjuvant for an IN vaccine that induces substantial protective immune responses against viral infections and tumor growth. ${ }^{19}$ The same research group also showed that a single nasal immunization with the NKT cell activation can induce safe and effective protection. ${ }^{20}$ However, potential cross-protection against variant influenza infections by a nasal vaccine that specifically activates NKT cells has not been demonstrated. Moreover, a precise NKT cell activation mechanism in IN immunization has not yet been clarified, a possibility that might have been overlooked previously owing to the extremely low abundance of NKT cells in the mucosa. ${ }^{21}$ In this study, we examined these issues and found that an NKT cell-mediated nasal vaccine can induce effective cross-protection against different strains of influenza virus. We have also established a unique mechanism for NKT cell activation in nasal tissue that contributes to the enhancement of mucosal IgA production.

\section{RESULTS \\ Cross-protective effect of intranasal influenza vaccine with $\alpha$-GalCer}

At first, we confirmed the mucosal adjuvant efficacy of $\alpha$-GalCer for influenza virus HA vaccine. We examined specific antibody production, HA-specific T-cell response, and residual virus titers following nasal virus challenge and found that the IN coadministration of $\alpha$-GalCer with HA vaccine effectively enhanced both humoral and cellular antigen-specific immunity in an NKT celldependent manner (Supplementary Figures 1 and 2). These results are fundamentally consistent with those reported by Ko et al. ${ }^{19}$

We then evaluated the cross-protective effect of IN immunization with $\alpha$-GalCer adjuvant against various influenza viruses. Mice received various HA vaccines with $\alpha$-GalCer adjuvant either IN or via intramuscular (IM) injection. In mice IN immunized with A/PR8 (H1N1) HA vaccine plus $\alpha$-GalCer, significant levels of anti-A/PR8 HA IgA and anti-A/PR8 HA IgG titer levels were detected in nasal and serum (respectively) as well as significant serum hemagglutination inhibition (HI) titer against $\mathrm{A} / \mathrm{PR} 8$ virus, resulting in the elimination of $\mathrm{A} / \mathrm{PR} 8$ virus in the nasal wash fluid following nasal live virus challenge (Figure 1a). Similar IN immunization with A/Yamagata (H1N1) vaccine plus $\alpha$-GalCer also induced markedly enhanced levels of nasal antiA/PR8 HA IgA and serum anti-A/PR8 HA IgG, even though no significant specific serum HI titer was induced. This heterologous immunization nonetheless enabled substantial elimination of A/PR8 challenge virus (Figure 1a). Mice IN immunized with A/Guizhou (H3N2) HA vaccine plus $\alpha$-GalCer exhibited a low response of nasal anti-A/PR8 HA IgA but no correspond- ing specific serum IgG or HI titer and low protective efficiency against A/PR8 virus challenge (Figure 1a). Antibody production, serum $\mathrm{HI}$ titer, and protection from virus challenge were not observed for IN immunizations with $\mathrm{B} / \mathrm{Ibaraki}$ or negative control (HA-free) vaccines (Figure 1a). When mice IN immunized with A/PR8 HA plus $\alpha$-GalCer were nasally challenged with various influenza viruses, results consistent with cross-protection were observed (Figure 1c) whereby the immunization induced significant levels of serum HI titer to both A/PR8 and A/Yamagata and, furthermore, caused an efficient elimination of both homologous (A/PR8) and heterologous (A/Yamagata) challenging viruses. Challenge infections with $\mathrm{A} / \mathrm{Guizhou}$ virus were slightly but significantly inhibited from the same immunization scheme, whereas no significant protection was observed for $\mathrm{B} / \mathrm{Ib}$ araki virus challenge.

In contrast, when the same HA vaccines with $\alpha$-GalCer were IM administered, no nasal IgA production was observed in any group (Figure 1b). Significant HI titer against A/PR8 virus was observed only in the group of homologous combination (A/PR8 HA vaccine), and inhibition of challenging virus was possible only in this group (Figure 1b).

To examine the cross-protective effects in influenza virusinduced lethal pneumonia, the IN or IM immunized mice were challenged with a lethal dose $\left(40 \mathrm{LD}_{50}\right)$ of $\mathrm{A} / \mathrm{PR} 8$ viruses exposed via the respiratory tract. All mice IN immunized with A/PR8 or A/Yamagata HA vaccine survived. Forty percentage of mice IN immunized with A/Guizhou HA vaccine survived at 14 days following the challenge and completely recovered thereafter (Figure 1a). No mice survived from the group immunized with B/Ibaraki HA or $\alpha$-GalCer alone at 14 days following the challenge (Figure 1a). These results are in contrast to the IM immunized mice, whereby only the group immunized with A/PR8 HA vaccine was strongly protected from A/PR8 challenge ( $100 \%$ survival). Only $20 \%$ of mice in the group receiving A/Yamagata HA IM vaccine survived (Figure 1b), and no survival was observed in the mice IM immunized with A/Guizhou HA vaccine, $B /$ Ibaraki HA vaccine, or $\alpha$-GalCer alone (Figure 1b). Taken together, these observations indicate that IN vaccination coadministered with $\alpha$-GalCer adjuvant generates significant cross-protection against heterosubtypic virus infection, which is not apparent via IM vaccination.

\section{Cross-protection to H5N1 influenza induced by the nasal vaccine with $\alpha$-GalCer}

To further examine the cross-protective potential induced by the NKT cell-mediated nasal vaccine against $\mathrm{H} 5 \mathrm{~N} 1$ influenza, we prepared an inactivated whole-virion vaccine of $\mathrm{H} 5 \mathrm{~N} 1 \mathrm{influ}$ enza with formalin-inactivated virus derived from a patient in Vietnam (see Methods). This form of vaccine possesses substantial antigenicity and, consistent with this fact, vaccine administered IN without $\alpha$-GalCer induced moderate levels of nasal IgA and serum IgG production (Figure 2a). Moreover, it reduced the virus titer after nasal live-virus infection and completely eliminated the virus in two out of five mice (Figure 2a). In the lethal pneumonia model, this vaccine can almost completely protect mice from homologous virus infection (Figure $2 \mathbf{b}$ ) but not from 


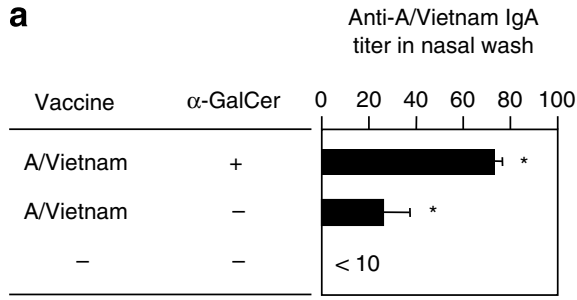

b

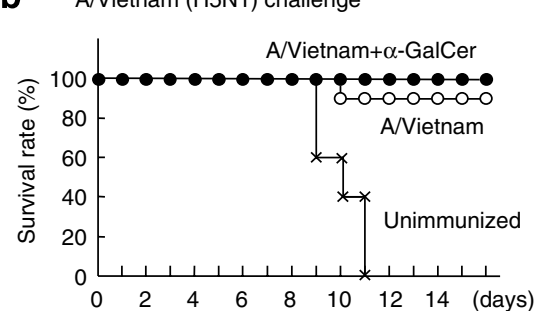

Anti-A/Vietnam IgG

titer in serum

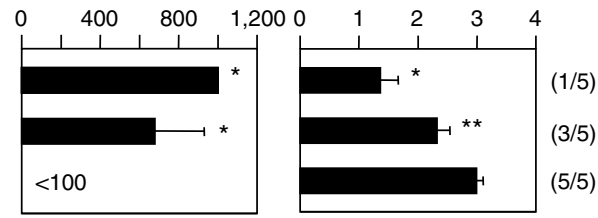

C

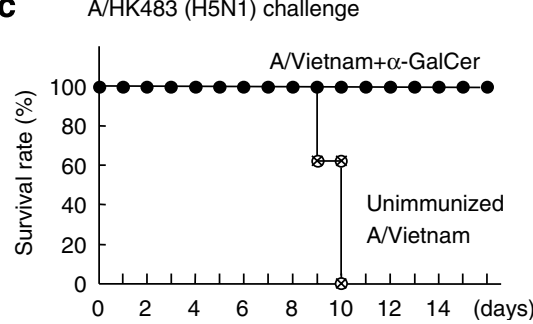

Figure 2 Cross-protection against H5N1 influenza. (a) Five mice per group were IN immunized with NIBRG14 (H5N1)-inactivated whole-virion vaccine with or without $\alpha$-GalCer. Two weeks after the second immunization, anti-A/Vietnam-specific nasal IgA and serum IgG titers were measured by end point ELISA. Another set of mice was IN infected with 1,000 PFU of A/Vietnam influenza virus in $1 \mu \mathrm{l} \mathrm{PBS} 2$ weeks after the second immunization. The nasal wash fluid was collected 3 days after virus challenge. The virus titer was measured by plaque assay. The values in parentheses indicate the numbers of mice failed to clear nasal viruses/total mice. All the experiments were repeated independently twice, with similar results. Each column represents the mean \pm s.d. ${ }^{*} P<0.01$ and ${ }^{* *} P<0.05$ vs. group of unimmunized mice. (b and $\left.\mathbf{c}\right)$ Survival curve of the mice immunized with $A / V i e t n a m$ whole-virion vaccine with $(\bullet)$ or without $(\bigcirc) \alpha$-GalCer after lethal A/Vietnam (b) or A/HK483 (c) virus challenge. The immunized or unimmunized $(x)$ mice were challenged with a lethal dose (1,000PFU in $20 \mu \mathrm{l})$ of virus at 2 weeks after the second immunization. The survival rate of the mice was then estimated daily. In c, unimmunized $(x)$ and A/Vietnam-immunized $(O)$ mice died with the same time course. ELISA, enzyme-linked immunosorbent assay; IN, intranasal; PBS, phosphate-buffered saline.

heterologous ones (A/HK483) (Figure 2c). On the other hand, when $\alpha$-GalCer was used as the adjuvant for this vaccine, the antibody production, particularly nasal IgA, was substantially increased (Figure 2a). When infected with live virus via nasal administration, the resulting nasal virus titer was markedly reduced; four out of the five mice completely eliminated the viruses (Figure 2a). Most importantly, this nasal vaccine with $\alpha$-GalCer caused complete protection from lethal pneumonia, induced not only by homologous but also by heterologous $\mathrm{H} 5 \mathrm{~N} 1$ influenza virus (Figure $\mathbf{2 b}, \mathbf{c}$ ). These results indicate that the nasal vaccine with $\alpha$-GalCer adjuvant can induce cross-protection against $\mathrm{H} 5 \mathrm{~N} 1$ influenza infection.

\section{Nasal activation of NKT cell is required}

We then investigated the importance of IN administration of $\alpha$-GalCer in this nasal vaccine. It is well known that $\alpha$-GalCer administration via the IV or intraperitoneal (IP) route will induce rapid cytokine production in the serum (Supplementary Figure 3A). However, IN administration of the same dose $(2 \mu \mathrm{g})$ of $\alpha$-GalCer induced only small amounts of IFN- $\gamma$ and IL-4 in the serum (Supplementary Figure 3A). Therefore, to examine the impact of IN administration of $\alpha$-GalCer in the nasal vaccine, we immunized mice with A/PR8 HA (IN) coadministered with $\alpha$-GalCer via either the IN or IP route. It is noteworthy that the antigen-specific nasal IgA and serum IgG production were not observed when $\alpha$-GalCer was administered IP (Supplementary Figure 3B). These data indicate that $\alpha$-GalCer must be coadministered with the antigen to effect a nasal vaccine that functions via NKT cell activation.

\section{Increase of NKT cell population in NALT and CLN after nasal vaccination}

To define the mechanism by which IN administration of $\alpha$-GalCer functions as a mucosal adjuvant, we examined how and where NKT cells were activated. The population of NKT cells in nasopharyngeal-associated lymphoid tissue (NALT) and regional cervical lymphnode (CLN) of naïve mice is normally very small $(0.04 \pm 0.01$ and $0.04 \pm 0.02 \%$, respectively, $n=3)$ (Figure 3). However, following IN administration of A/PR8 $\mathrm{HA}$ and $\alpha$-GalCer, the NKT cell population in those tissues was increased with a peak at 4 days post-immunization $\left(0.18 \pm 0.01^{*}\right.$ and $0.20 \pm 0.01 \%{ }^{\star}$, respectively, ${ }^{\star} P<0.05$ vs. naive) (Figure 3 ). Different from the case of IP or IV $\alpha$-GalCer injection, ${ }^{22}$ the nasal NKT cells did not reduce the level of TCR after the IN immunization (data not shown). The same vaccinations did not alter NKT cell population in the spleen throughout 1 week postimmunization (at 4 days, naive $2.34 \pm 0.25 \%$ vs. IN immunized $2.36 \pm 0.34 \%$, not significant) (Figure 3). These results suggest that the nasal vaccine with $\alpha$-GalCer mainly influences local but not systemic NKT cells.

We further investigated the distribution of IN administered $\alpha$-GalCer. To this end, we prepared Cy3-labeled $\alpha$-GalCer (Supplementary Figure 4), which was then IN administered to $\mathrm{BALB} / \mathrm{c}$ mice followed by examination of the number of Cy3-positive cells in NALT, CLN, and the spleen. The data shown in Figure 4a indicate that the distribution of IN administered $\alpha$-GalCer was primarily limited to NALT and CLN, whereas only a small fraction transiently reached the spleen. Cy3-positive cells were hardly detected in the liver (data not 


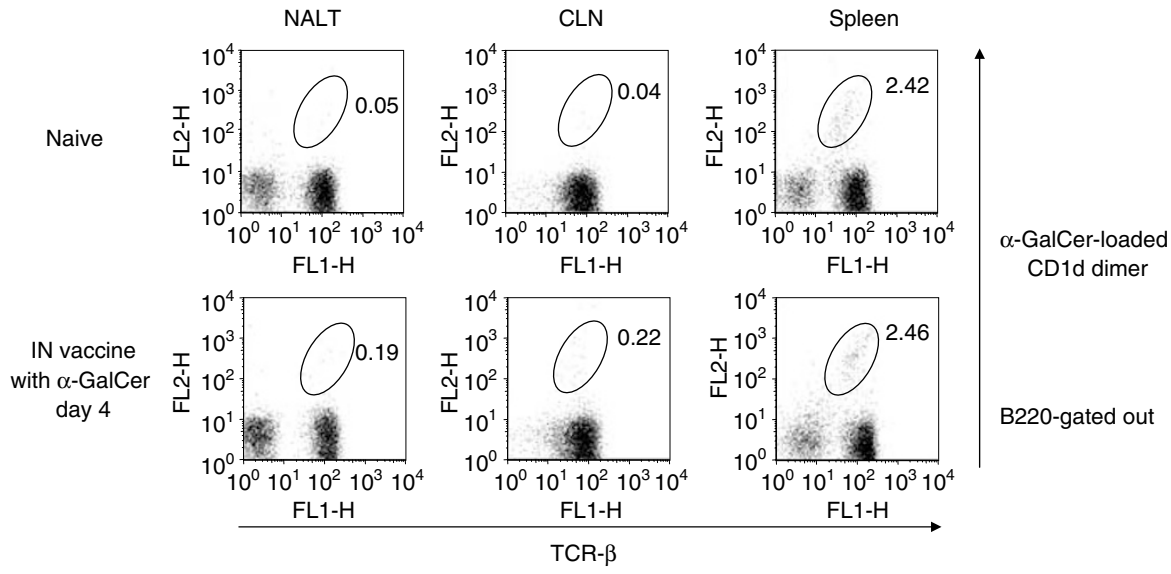

Figure 3 Increase of NKT cell population in NALT and CLN after the nasal vaccination with $\alpha$-GalCer. BALB/c mice were IN immunized with A/PR8 HA vaccine and $\alpha$-GalCer. Naive and immunized mice were killed, and NKT cell population in NALT, CLN, and spleen was evaluated with FACS. The samples were costained with anti-B220 mAb, and the B220+ cells were electronically gated out to exclude nonspecific staining and to enhance NKT cell population in the fluorescence-activated cell sorter dot plot pattern. Upper panels: representative FACS data of naive and immunized mice (4 days post-immunization). The inset numbers are a percentage of the gated region of the samples. Similar results were obtained from three independent experiments. CLN, cervical lymphnode; HA, hemagglutinin; IN, intranasal; NALT, nasopharyngeal-associated lymphoid tissue; NKT, natural killer T cell.

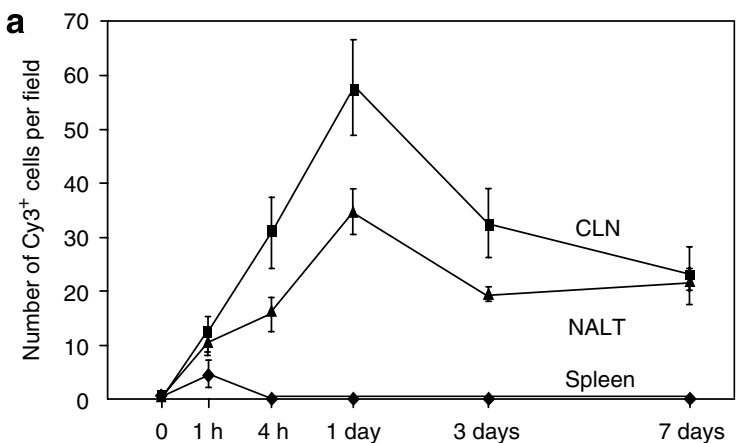

b
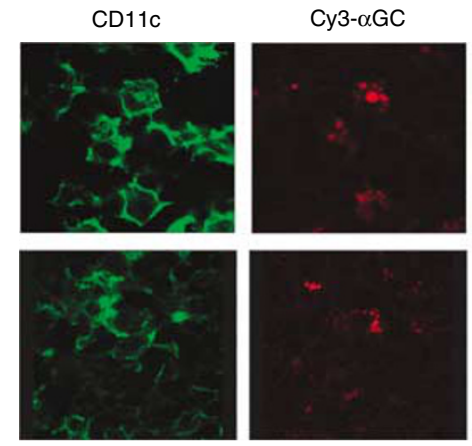

C

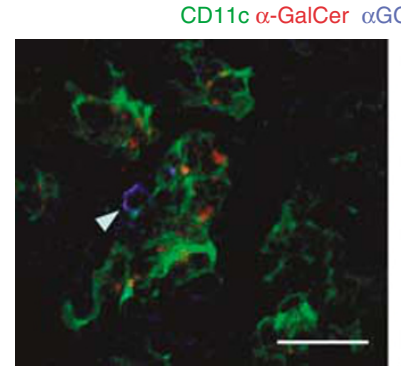

CD11c $\alpha$-GalCer $\alpha G C$-unloaded CD1d dimer Day 4

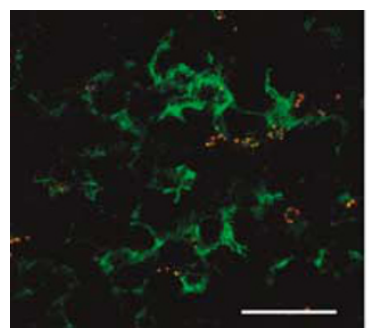

CD11c $\alpha G C-l o a d e d$ CD1d dimer Naive

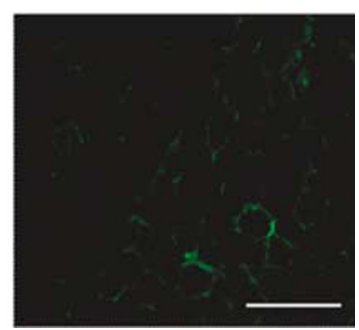

Figure 4 In vivo distribution of IN administered $\alpha$-GalCer. BALB/c mice were IN administered with Cy3-labeled $\alpha$-GalCer (Supplementary Figure 4). (a) NALT, CLN, and spleen were collected from three different individuals at each time point. The Cy $3^{+}$cells per field (original magnification $\times 630$; $0.0125 \mathrm{~mm}^{2}$ ) were counted, and the mean \pm s.d. of at least five different fields is shown. (b) At day 3, NALTs and CLNs were stained with anti-CD11c mAb, followed by FITC-conjugated anti-hamster IgG. Representative confocal microscopic pictures are shown. Bar $=8 \mu \mathrm{m}$. Original magnification $\times 630$. (c) NALT samples were stained with anti-CD11 c mAb, followed by FITC-conjugated anti-hamster IgG. The specimens were further stained with biotinylated CD1d dimer with or without loading of $\alpha$-GalCer, followed by Cy5-conjugated streptavidin. Upper panels: two representative confocal microscopic pictures of NALT at day 4 for $\alpha$-GalCer-loaded CD1d dimer staining. Lower panels: negative controls with $\alpha$-GalCer-unloaded CD1d dimer staining at day 4 (left) and $\alpha$-GalCer-loaded CD1d dimer staining of NALT samples from naive mice (right). Bar $=20 \mu \mathrm{m}$. The arrow heads represent NKT cells. CLN, cervical lymphnode; FITC, fluorescein isothiocyanate; IN, intranasal; NALT, nasopharyngeal-associated lymphoid tissue; NKT, natural killer T cell.

shown). At 7 days following IN administration, the Cy3-positive cells remained in the NALT and CLN whereas none were detectable in the spleen.
Dendritic cells (DCs) are known to strongly stimulate NKT cells. We therefore examined whether DCs in NALT and CLN take up IN administered $\alpha$-GalCer. NALT and CLN tissues from 
a

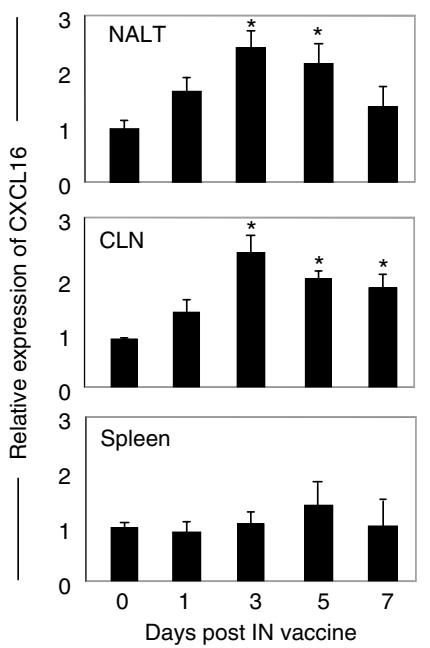

b

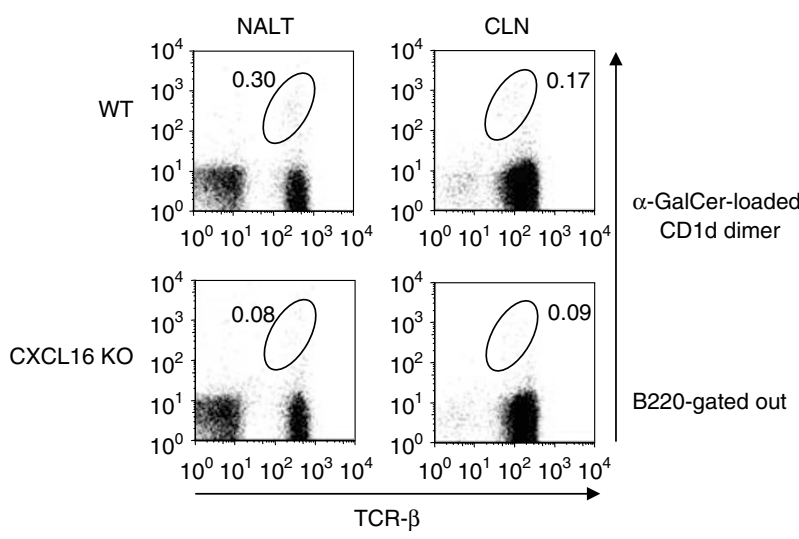

C

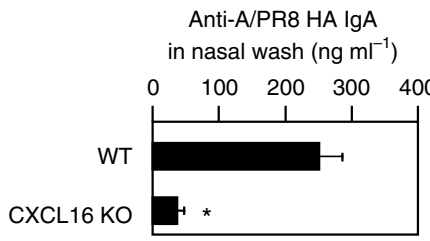

Anti-A/PR8 HA IgG in serum $\left(\mu \mathrm{g} \mathrm{ml}^{-1}\right)$

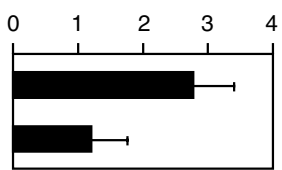

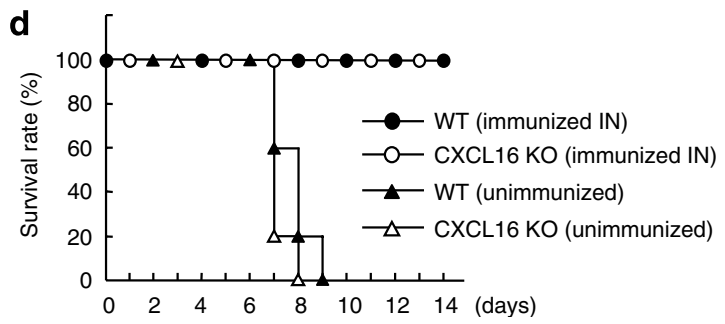

Figure 5 Involvement of CXCL16/CXCR6 in the increase of NKT cells after nasal vaccination with $\alpha$-GalCer. (a) Expression of CXCL16 after the vaccination. After IN immunization with A/PR8 HA vaccine and $\alpha$-GalCer, expression of CXCL16 in NALT, CLN, and spleen was examined with realtime PCR. Each bar represents the mean \pm s.d. of triplicate wells from four pooled samples. ${ }^{*} P<0.01 \mathrm{vs}$. day 0 . Similar results were obtained from two independent experiments. (b) Reduced increase of NKT cell population in CXCL16 KO mice. WT and CXCL16 BALB/C mice were IN immunized with A/PR8 HA vaccine and $\alpha$-GalCer. Four days later, the immunized mice were killed, and NKT cell population in NALT and CLN was evaluated with fluorescence-activated cell sorter as in Figure 3. Similar results were obtained from three independent experiments. (c) Antibody production in CXCL16 KO mice. WT and CXCL16 KO BALB/c mice were IN vaccinated with A/PR8 HA and $\alpha$-GalCer and the antigen-specific IgA in nasal wash and IgG in serum were measured. Each column represents the mean \pm s.d. of five mice. ${ }^{\star} P<0.01$ vs. WT. (d) Lethal infection in CXCL16 KO mice. WT and CXCL16 KO mice (five per group) were IN immunized or left unimmunized and challenged with a lethal dose of A/PR8 virus as in Figure 1. The survival rate of the mice was then estimated daily. Similar results were obtained from two independent experiments. CLN, cervical lymphnode; HA, hemagglutinin; IN, intranasal; NALT, nasopharyngeal-associated lymphoid tissue; NKT, natural killer T cell.

treated animals were stained using anti-CD11c mAb. Control hamster IgG showed no specific staining (not shown). We found that $\mathrm{CD} 11 \mathrm{c}^{+}$cells appeared as some clusters mainly in the perifollicular zone (not shown). The representative photograph shown in Figure $\mathbf{4 b}$ indicates that IN administered Cy3labeled $\alpha$-GalCer was taken up by the DCs and stored in the intracellular vesicles. We further stained the same tissues with anti-F4/80, TCR $\beta$, and B220 mAbs, but were unable to find stain overlap with Cy3-labeled $\alpha$-GalCer (Supplementary Figures 5 and 6). These data indicate that the IN administered $\alpha$-GalCer was mainly taken up by DCs that are locally distributed in NALT and its draining LN rather than by systemic DCs. Furthermore, we also stained these tissues with $\alpha$-GalCer-loaded or unloaded CD1d dimer. In this experiment, we could successfully visualize colocalization of NKT cells and $\alpha$-GalCer-storing DCs (Figure 4c, upper panels). Staining with $\alpha$-GalCer-unloaded CD1d dimer showed no signal for NKT cells (Figure 4c, lower left panel). In naive NALT tissues, we could hardly detect signals for NKT cells (Figure 4c, lower right panel), reflecting their very low frequency in naive state (cf. Figure 3). These data confirm the increase of NKT cells and the interaction between NKT cells and DCs in the nasal tissues following IN administration of $\alpha$-GalCer.

\section{Involvement of CXCL16/CXCR6 in the increase of NKT cells in NALT and CLN}

We have previously demonstrated that NKT cells strongly express a chemokine receptor CXCR6 and that an interaction with its exclusive ligand, CXCL16, is involved in the trafficking of NKT cells. ${ }^{23}$ Therefore, we investigated whether CXCL16/ CXCR6 mechanisms also contribute to the increased population of NKT cells following nasal vaccination with $\alpha$-GalCer. Using real-time PCR, we first examined the expression of CXCL16 in NALT, CLN, and spleen before and after nasal vaccination. We found that the expression of CXCL16 was upregulated only in NALT and CLN but not in the spleen, with a peak at day 3 post-vaccination (Figure 5a). Subsequently, we used CXCL16deficient (CXCL16 knockout (KO)) mice to confirm the role of CXCL16. We vaccinated WT and CXCL16 KO mice IN and then examined the NKT cell population in NALT and CLN. Four days post-vaccination, the NKT cell population in these tissues increased in WT mice $(0.28 \pm 0.06 \%, n=3)$, whereas 
that in CXCL16 KO mice did not reach the level of WT mice $(0.09 \pm 0.02 \%, P<0.01)$ (Figure $5 \mathbf{b})$. The NKT cell populations in NALT and CLN of naive CXCL16 KO mice $(0.04 \pm 0.02 \%$ for NALT and $0.06 \pm 0.02 \%$ for CLN, $n=3$, respectively) were not markedly different from those in naive WT mice (cf. Figure 3 ), and the time course of the NKT cell change was not different between WT and CXCL16 KO mice (not shown). These results clearly demonstrate that the increase in NKT cells induced by nasal vaccine with $\alpha$-GalCer is substantially impaired in the absence of CXCL16.

Furthermore, we investigated the impact of CXCL16 on antigenspecific antibody production. We vaccinated WT and CXCL16 KO mice IN and measured the antibody production. We found that the production of mucosal IgA but not serum IgG in CXCL16 KO mice was significantly lower than in WT mice (Figure 5c), indicating that the CXCL16-mediated NKT cell increase in NALT and CLN is necessary for stimulating secretary IgA production. In lethal influenza challenge, however, IN immunized CXCL16 KO mice demonstrated complete survival as WT mice did (Figure 5d). This result suggests the existence of a compensatory mechanism in vivo against influenza infection as discussed below.

\section{Enhancement of secretary IgA production by NKT cell activation, which requires IL-4}

The hallmark of mucosal immunity is the production of secretary IgA. Previous reports have indicated that NKT cells induce B-cell activation and antibody production, ${ }^{24,25}$ but the mechanism of enhancement of secretary IgA production of NKT cells has not been demonstrated. In view of this, we further examined the contribution of cytokines produced by $\alpha$-GalCer-stimulated NKT cells on IgA production. We first used in vitro IgA production assay on tissues derived from IL-4-deficient or IFN- $\gamma$-deficient mice in a C57BL/ 6 background. CLN tissue from the animals was obtained before and after the nasal vaccination and stimulated with IL-5 plus transforming growth factor- $\beta$ in the presence or absence of $\alpha$-GalCer. In the experimental condition employed here, the addition of IL-5 plus transforming growth factor- $\beta$ only slightly upregulated the IgA production by CLN derived from either naive or IN vaccinated mice (Figure 6a). The addition of $\alpha$-GalCer to the culture of CLN from IN vaccinated WT mice substantially upregulated IgA production (Figure 6a, WT). This upregulation appears to be entirely dependent upon IL- 4 because CLN derived from IN vaccinated IL-4 KO mice demonstrated no enhancement of IgA production, regardless of the presence of $\alpha$-GalCer (Figure 6a, IL-4 KO). By contrast, in the culture of CLN from IN vaccinated IFN- $\gamma$ KO mice, the increase of IgA production seen in WT was enhanced even further (Figure 6a, IFN- $\gamma \mathrm{KO}$ ). Interestingly, the IgA production enhanced by the addition of $\alpha$-GalCer was totally dependent on the increase of NKT cells in CLN after the nasal vaccination; it was not observed when using CLN derived from naive mice (Figure 6a), in which very few NKT cells were found (cf. Figure 3). a

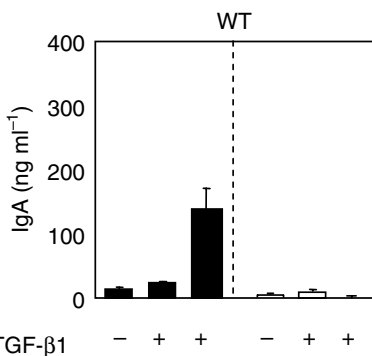

$\alpha$-GalCer
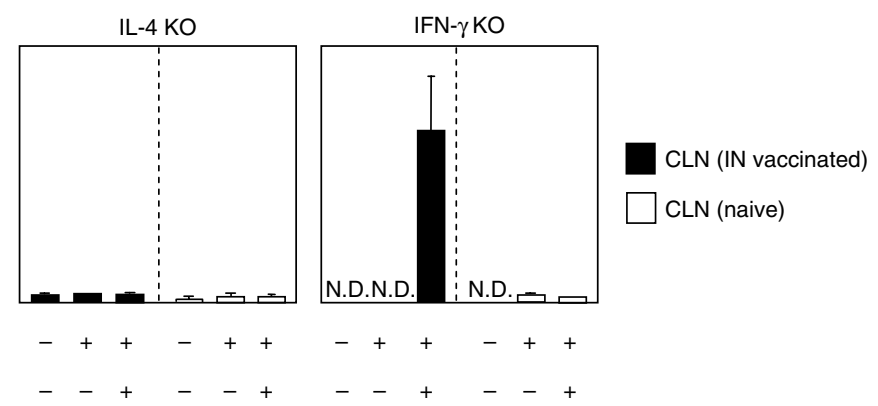

b

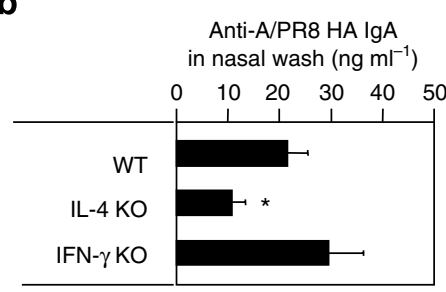

Anti-A/PR8 HA IgG

in serum $\left(\mu \mathrm{g} \mathrm{ml}^{-1}\right)$

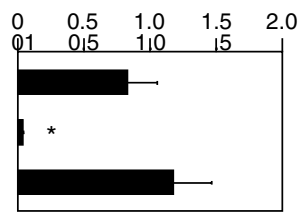

C

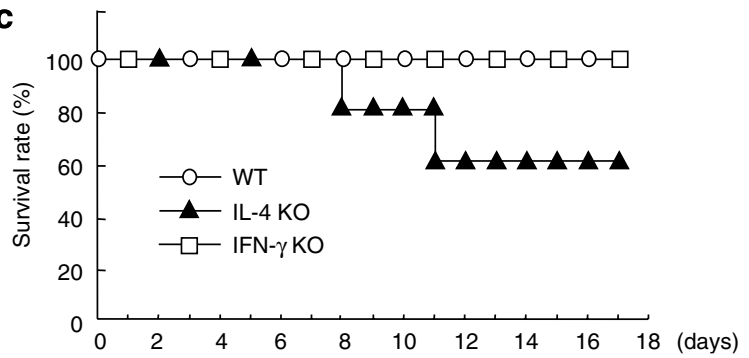

Figure 6 Involvement of NKT cell-mediated cytokines in the production of IgA. (a) WT, IL-4 KO, and IFN- $\gamma$ KO B6 mice were IN immunized with A/PR8 HA and $\alpha$-GalCer. Four days after the immunization, single-cell suspensions $\left(2 \times 10^{5}\right.$ per well) from CLN of these mice were stimulated with recombinant mouse IL-5 (10 $\left.\mathrm{ng} \mathrm{ml}^{-1}\right)$ and human transforming growth factor- $\beta 1\left(1 \mathrm{ng} \mathrm{ml}^{-1}\right)$ in the presence or absence of $\alpha$-GalCer $\left(200 \mathrm{ng} \mathrm{ml}^{-1}\right)$. Six days later, the IgA production in the culture supernatants was determined with ELISA. CLNs from naive mice were also cultivated in the same manner. The data represent the mean \pm s.d. of triplicate wells. Representative data from three independent experiments with similar results. (b) In vivo antibody production. WT, IL-4 KO, and IFN- $\gamma$ KO B6 mice were IN vaccinated with A/PR8 HA and $\alpha$-GalCer, and the antigen-specific IgA in nasal wash and IgG in serum were measured as in Figure 1. Each column represents the mean \pm s.d. of five mice. ${ }^{*} P<0.01$ vs. WT. (c) Protection from lethal infection. WT, IL-4 KO, and IFN- $\gamma$ KO B6 mice (five per group) were IN immunized and challenged with a lethal dose of A/PR8 virus as in Figure 1. The survival rate of the mice was then estimated daily. Similar results were obtained from two independent experiments. Unimmunized mice were all dead within 7 days (not shown). CLN, cervical lymphnode; ELISA, enzyme-linked immunosorbent assay; HA, hemagglutinin; IFN- $\gamma$, interferon- $\gamma$; IL-4, interleukin; IN, intranasal; NKT, natural killer T cell. 
When these mice were IN vaccinated, antigen-specific mucosal IgA production (as well as IgG production in serum) was significantly decreased in IL- $4 \mathrm{KO}$ but not in IFN- $\gamma \mathrm{KO}$ mice compared with WT mice (Figure $\mathbf{6 b}$ ). When IN vaccinated WT or IFN- $\gamma \mathrm{KO}$ mice were challenged with a lethal dose of influenza virus, all the mice survived (Figure $\mathbf{6 c}$ ). In contrast, $40 \%$ of IN vaccinated IL- $4 \mathrm{KO}$ mice died by day 11 after the infection (Figure 6c). Unimmunized mice were all dead within 7 days (not shown).

Taken together, these results suggest that the NKT cell increase following nasal vaccination with $\alpha$-GalCer is (a) partly mediated via interaction of CXCL16/CXCR6 and that it is (b) important or required for significant secretary IgA production. Furthermore, IL-4 induced by NKT cell activation plays a role in enhancing IgA production, but IFN- $\gamma$ does not play such a role.

\section{DISCUSSION}

The results of this study demonstrated that $\alpha$-GalCer is an effective mucosal adjuvant for the induction of cross-protection from different strains of influenza viruses. We also presented mechanisms of NKT cell activation when a specific glycolipid ligand, $\alpha$-GalCer, is administered to nasal mucosa. Regarding the development of a vaccine for pandemic influenza, predicting the correct virus strain before an outbreak remains a key issue. The United States Food and Drug Administration has recently approved an $\mathrm{H} 5 \mathrm{~N} 1$ vaccine, but significant concerns exist that the antigens used may not be sufficiently homologous to an outbreak strain. ${ }^{26}$ Accordingly, cross-protection induced by a nasal vaccination with NKT cell activation, as contemplated herein, would broaden the much-needed effectiveness of such a vaccine, even if immunization antigens and outbreak strain are not homologous. The mechanisms described in this study serve to strengthen the rationale of a mucosal vaccine with NKT cell activation and enhance the development of a novel mucosal vaccine not only for influenza but also for various other diseases.

Although the precise mechanism of cross-protection induced by mucosal vaccine remains unclear, an involvement of mucosal IgA has been demonstrated. ${ }^{10-12}$ In fact, we observed that the cross-protective effect of NKT cell-mediated vaccines was induced only when it was administered IN, but not IM, and only when substantial amounts of nasal IgA were produced (Figure 1). HI assays suggest the possibility that nasal IgA is more important than serum IgG for induction of cross-protection because some cross-protection was still apparent even when serum HI titer was not detected (Figure 1a, A/Yamagata- and A/Guizhou-vaccinated group). Accordingly, measuring the titer of antigen-specific nasal IgA may be a useful way to evaluate cross-reactive protection against influenza viruses following mucosal vaccination. However, the overall titers of antibodies (nasal IgA and serum IgG) also appear to be important because a more effective cross-protection was observed when a higher titer of both antibodies was produced (Figure 1). It is still possible that serum IgG plays a supportive role for nasal IgA to induce effective cross-protection.

The nasal vaccination with $\mathrm{A} /$ Vietnam vaccine plus $\alpha$ GalCer induced a clear cross-protection against A/HK483 virus challenge (Figure 2c). Although the A/Vietnam and A/HK483 influenza viruses are both of subtype $\mathrm{H} 5 \mathrm{~N} 1$, significant pathogenic heterogenicity exists between them. This was demonstrated by the mice that were IN immunized with A/Vietnam vaccine (no adjuvant) and challenged with $\mathrm{A} / \mathrm{HK} 483$ and subsequently died at the same rate as unimmunized, challenged mice (Figure 2c). In strong contrast, A/Vietnam vaccination coadministered with $\alpha$-GalCer completely protected mice from A/HK483 challenge. The A/Vietnam vaccination with $\alpha$-GalCer adjuvant induced a significantly higher level of nasal IgA production than the same vaccination alone (Figure 2a), which is consistent with the idea that a high titer of secretory IgA is important for the induction of effective cross-protection.

The population of NKT cells in NALT and CLN is very small in a naive (unimmunized) state. Therefore, we first anticipated that IN administered $\alpha$-GalCer would reach some distant tissues that generally contain higher concentrations of NKT cell populations, such as the spleen or liver, and activates the NKT cells in those organs. However, this mechanism seems unlikely in view of our present data because IN administered $\alpha$-GalCer was detected only slightly in those organs (Figure 4). Furthermore, although IP administered $\alpha$-GalCer induces rapid activation of splenic or hepatic NKT cells, ${ }^{22}$ it exerted no measurable adjuvant effect for an IN administered vaccine (Supplementary Figure 3). These results indicate that the activation of nasal NKT cells (including those in regional $\mathrm{LN}$ ) is a necessary requirement for induction of the adjuvant effect, whereby an NKT cell activator, such as $\alpha$-GalCer, is coadministered IN with a vaccine antigen. Recently, Ko et al. ${ }^{19}$ reported that DCs in mediastinal LN but not CLNs are mainly activated by IN administration of $\alpha$-GalCer. This discrepancy could be explained by the difference in volume of vaccine IN administered: they administered $10 \mu$ into each nostril (20 $\mu$ l per mouse), ${ }^{19}$ whereas we administered $5 \mu \mathrm{l}$ per mouse in total. The higher volume of vaccine might reach more distant regions.

We have previously demonstrated that CXCL16/CXCR6 interaction is important for in vivo trafficking of NKT cells and for exhibiting their immunoregulatory effect. ${ }^{23}$ In the nasal vaccine with $\alpha$-GalCer adjuvant, CXCL16/CXCR6 may also play an important role by increasing the concentration of nasal NKT cells. In the present study, such a localized increase of NKT cells appeared necessary for the induction of IgA production (Figures 5 and 6). However, the immunized CXCL16 KO mice exposed to lethal challenge infection demonstrated complete survival (Figure 5d). This may be due to the lower but significant levels of nasal IgA (30-40 $\left.\mathrm{ng} \mathrm{ml}^{-1}\right)$ and serum $\operatorname{IgG}\left(1.0-1.5 \mu \mathrm{g} \mathrm{ml}^{-1}\right)$ that were produced in those animals. These antibody levels were comparable with those seen in A/Yamagata IN immunized mice, which were also completely resistant to A/PR8 challenge (Figure 1a). Therefore, it is possible that CXCL16 is important for the recruitment of NKT cells and NKT cell-mediated IgA production, whereas the lack of CXCL16 causes a diminished efficiency of this IN immunization mechanism, but it is not low enough to disrupt challenge protection in this model. Similarly, although antibody production (both nasal IgA and serum IgG) was significantly impaired in IL-4 KO mice (Figure 6b), more 
than half of the mice survived upon lethal influenza infection (Figure 6c). Because host defense is mediated by multiple, sometimes overlapping, mechanisms including both innate and acquired immunity, as well as humoral and cellular immunity, there is potential for the lack of one mechanism in this knockout model to be at least partially compensated by others. Consistent with this possibility, it has previously been reported that unimmunized NKT KO mice exhibit similar resistance against challenge infection as unimmunized WT mice, ${ }^{27}$ presumably because alternate host immune mechanisms can be recruited to cover the NKT cell deficiency. Taken together, we suggest that although NKT cells are required for this proposed method of nasal vaccination with $\alpha$-GalCer adjuvant (Supplementary Figure 1), the cells may not be absolutely required to generate at least some resistance to natural influenza infection.

Prophylactic agents, including vaccine, should have sufficient safety for clinical use. This is anticipated to be of less concern with the $\alpha$-GalCer IN vaccination model because the human safety of this compound has been examined previously. Patients with solid tumors received $\alpha$-GalCer $\left(50-4800 \mu \mathrm{g} \mathrm{m}^{-2}\right)$ IV, as a proposed immunotherapy with no significant adverse effects reported. ${ }^{28}$ Although the safety profile of $\alpha$-GalCer when administered IN to humans needs further examination, the previous human trial of the compound administered at fairly high doses using IV protocols strongly encourages the development of NKT-activating nasal vaccines for humans with $\alpha$-GalCer or other glycolipid adjuvants. Further clinical studies with appropriate safety methodology will be necessary to clarify these issues.

\section{METHODS}

Vaccines and influenza viruses. Hemagglutinin vaccines (split-product virus vaccines) were prepared from influenza viruses, including A/Puerto Rico/8/34 (A/PR8; H1N1), A/Yamagata/120/86 (A/Yamagata; H1N1), A/Guizhou/54/89 (A/Guizhou; H3N2), and B/Ibaraki/2/85 (B/Ibaraki) strains, as previously described. ${ }^{29}$ The $\mathrm{H} 5 \mathrm{~N} 1$ vaccine used in this study was a formalin-inactivated whole-virion vaccine, NIBRG14. NIBRG14 was derived from a recombinant avirulent avian virus containing modified HA and neuraminidase from the highly pathogenic avian influenza strain A/Vietnam/1194/2004 virus and other viral proteins from the influenza strain A/PR/8/34 (H1N1).$^{30}$ Modified HA lacks the multibasic amino acids at the cleavage site. The A/PR8 virus used for the challenge experiments was adapted to mice by subculturing 148 times in ferrets, 596 times in mice, and 73 times in 10-day-old fertile chicken eggs. The strains of $\mathrm{H} 5 \mathrm{~N} 1$ virus for the challenge experiments were A/Vietnam/1194/04 (A/VN/1194/04, H5N1) and A/Hong Kong/483/97 (A/HK/483/97, H5N1).

Reagents. Cholera toxin B subunits containing a trace amount of holotoxin $\left(\mathrm{CTB}^{\star}\right)$ were prepared by adding $0.1 \% \mathrm{CT}$ (holotoxin) to CT B subunit obtained from Sigma (St. Louis, MO). $\alpha$-GalCer was synthesized in our laboratory. Cy3-labeled $\alpha$-GalCer was synthesized as indicated in Supplementary Figure 3.

Immunization. BALB/c mice (6- to 8-week-old) were purchased from Japan Clea (Tokyo, Japan). NKT cell-lacking J $\alpha 281$ gene-deficient (NKT $\mathrm{KO})$ mice were described previously. ${ }^{31}$ CXCL16 KO mice were described previously. ${ }^{32}$ Both kinds of KO mice were backcrossed to BALB/c mice more than nine generations before the experiments. All animal experiments were carried out in accordance with the guidelines for animal experiments of the National Institute of Infectious Diseases, Japan and RIKEN Research Institute for Allergy and Immunology, Japan.

Five mice for each experimental group were anesthetized with diethyl ether and IN immunized primarily by dropping a total $5 \mu$ of phosphatebuffered saline (PBS) containing $1 \mu \mathrm{g}$ of various HA vaccines with $2 \mu \mathrm{g}$ of $\alpha$-GalCer or Cholera toxin B subunits containing a trace amount of holotoxin to each nostril. For IM immunization, the same amounts of HA vaccines and adjuvants were injected into the femoral muscle. Four weeks later, they were reimmunized in the same manner and left for 2 more weeks before assays. In some experiments, only $\alpha$-GalCer was IP administered.

Antivirus antibodies. Serum and nasal wash fluid were collected from the immunized mice for measurement of Abs. The levels of A/PR8specific IgA and IgG Abs were determined by enzyme-linked immunosorbent assay (ELISA), by using a twofold serial dilution of either purified HA-specific IgA (320 $\left.\mathrm{ng} \mathrm{ml}^{-1}\right)$ or IgG $\left(160 \mathrm{ng} \mathrm{ml}^{-1}\right)$ as a standard, as described previously., ${ }^{73}$ To determine the level of IgA and IgG Abs against $\mathrm{A} /$ Vietnam virus ( $\mathrm{H} 5 \mathrm{~N} 1)$, an end point ELISA was performed using plates coated with the formalin-inactivated whole virus used for the vaccination (NIBRG14). End point titers that were twofold higher than those of unimmunized control sera were considered positive. Before the HI tests, receptor-destroying enzyme (RDE(II); Denka Seiken Ltd., Tokyo, Japan) was added to the red blood cell-treated sera to inactivate nonspecific hemagglutination inhibitors at $37^{\circ} \mathrm{C}$ overnight followed by incubation at $56^{\circ} \mathrm{C}$ for $1 \mathrm{~h}$ to inactivate receptor-destroying enzyme. HI tests were performed according to the microtiter method of Sever. ${ }^{34}$ Briefly, aliquots of $25 \mu \mathrm{l}$ of serial twofold dilutions of the treated serum samples were mixed with $4 \mathrm{HA}$ units of virus in microtiter plates and incubated at room temperature for $60 \mathrm{~min}$. Then, $50 \mu \mathrm{l}$ of $0.5 \%$ chicken red blood cells was added to each well and incubated at room temperature for 30-40 min. The HI titer was expressed as the reciprocal of the highest serum dilution that completely inhibited hemagglutination of 4 HA units of the virus.

Virus challenge, measurement of the virus titer, and protection from lethal pneumonia. For IN virus titer measurement, each mouse was infected by IN administration of $1 \mu \mathrm{l}$ PBS containing virus suspension with 1,000 PFU of the indicated virus at 2 weeks after the second immunization, according to a modification of the procedure of Yetter and coworkers. ${ }^{35,36}$ As $1 \mu$ l of the virus suspension remained in the local nasal area and could not enter the lung tissue, the initial viral infection was limited to the nasal area. Three days after the nasal challenge, the nasal wash and serum specimens were collected for virus and $\mathrm{Ab}$ titration. The virus titer was measured as previously described using Madin-Darby canine kidney cells. ${ }^{29}$ To examine the protective effect against influenza virus-induced lethal pneumonia, mice were challenged with 1,000 PFU of A/PR8 $\left(40 \mathrm{LD}_{50}\right), \mathrm{A} /$ Vietnam, or A/HK483 virus in $20 \mu \mathrm{l} \mathrm{PBS}$. One thousand PFU of A/Vietnam virus in $10 \mu \mathrm{l} \mathrm{PBS}$ represented $63 \mathrm{LD}_{50}$, and the IN administration of $20 \mu \mathrm{l}$ volume permits the virus to reach the lower respiratory tract, which increases the lethality. The body weight and survival rate of the mice were then estimated daily.

FACS analysis and real-time PCR. Single-cell suspensions were prepared from NALTs, CLNs, and spleens. For fluorescence-activated cell sorter, these cells were stained with fluorescein isothiocyanate (FITC)conjugated anti-TCR $\beta \mathrm{mAb}$ (clone; H57-597), phycoerythrin-labeled, $\alpha$-GalCer-loaded CD1d dimer, and allophycocyanin-conjugated antiB220 mAb (clone; RA3-6B2). These cells were analyzed using FACS Calibur (BD Bioscience, San Jose, CA) and FlowJo software (Tree Star, Ashland, OR). For real-time PCR, total RNA was extracted from these cells, and cDNA was synthesized using kits (QIAGEN, Valencia, CA) according to the manufacturer's instructions. Real-time quantitative PCR was performed using Applied Biosystems 7900HT Fast (Applied Biosystems, Foster City, CA) with TaqMan probes (Applied Biosystems) for mouse CXCL16 and G3PDH. 
Histological examination. Two micrograms of Cy3-labeled $\alpha$-GalCer in $10 \mu \mathrm{l}$ PBS was IN administered to BALB/c mice, and subsequently NALTs, CLNs, and spleens were harvested at different time points. The wholemount specimens were fixed with $4 \%$ paraformaldehyde (Cytofix; BD Bioscience) for $30 \mathrm{~min}$ at $4^{\circ} \mathrm{C}$ and then treated with Cytoperm washing solution (BD Bioscience) for membrane permeabilization. After Fc blocking with $10 \mu \mathrm{g} \mathrm{ml}^{-1}$ anti-CD16/CD32 mAb (clone 93; eBiosciences, San Diego, CA) in TSA blocking buffer (Perkin Elmer, Boston, MA), the specimens were stained with $5 \mu \mathrm{g} \mathrm{ml}^{-1}$ purified anti-CD11c (HL3) or TCR $\beta$ (H57-597) $\mathrm{mAb}$ (BD Bioscience) for $16 \mathrm{~h}$ at $4^{\circ} \mathrm{C}$. The specific binding of primary antibodies was detected with FITC-conjugated anti-hamster IgG (Jackson ImmunoResearch, West Grove, PA). The specimens were further treated with $5 \mu \mathrm{g} \mathrm{ml}^{-1}$ biotinylated anti-B220 (RA3-6B2; BD Bioscience) or F4/80 (BM8; eBiosciences) $\mathrm{mAb}$ for $2 \mathrm{~h}$ at $4^{\circ} \mathrm{C}$, followed by Cy5-conjugated streptavidin (Jackson ImmunoResearch). Isotype-matched controls confirmed each staining condition. To detect interaction between NKT cells and DCs, the whole specimens of NALT were pretreated as described above, stained with purified anti-CD11 c mAb, followed by FITC-conjugated anti-hamster IgG. The specimens were further stained with biotinylated CD1d dimer (BD Bioscience) with or without loading of $\alpha$-GalCer for $16 \mathrm{~h}$ at $4^{\circ} \mathrm{C}$, followed by Cy5-conjugated streptavidin. The specimens were analyzed with a DM-IRE2 confocal laser scanning microscope and Leica confocal software (Leica Microsystems, Tokyo, Japan).

In vitro IgA production. Seven-week-old C57BL/6 (B6) mice were purchased from Japan Clea. IL-4 KO and IFN- $\gamma$ KO mice (B6 background) were previously described. ${ }^{37,38} \mathrm{~A} / \mathrm{PR} 8 \mathrm{HA}$ and $\alpha$-GalCer were once IN administered as "immunization" to the mice (age-matched). Before or 4 days after the immunization, CLNs were harvested and single-cell suspensions were prepared. These cells $\left(2 \times 10^{5}\right.$ per well $)$ were stimulated with recombinant mouse IL-5 (10 $\left.\mathrm{ng} \mathrm{ml}^{-1}\right)$ and human transforming growth factor- $\beta 1\left(1 \mathrm{ng} \mathrm{ml}^{-1}\right)$ (both R\&D Systems, Minneapolis, MN) in the presence or absence of $\alpha$-GalCer $\left(200 \mathrm{ng} \mathrm{ml}^{-1}\right)$ in RPMI-1640 medium (Sigma) supplemented with $10 \%$ heat-inactivated fetal calf serum. Six days later, the culture supernatants were harvested and concentration of IgA was examined using a kit (Bethyl Lab., Montgomery, TX).

Serum cytokines. Two micrograms of $\alpha$-GalCer was IP or IN administered to $\mathrm{BALB} / \mathrm{c}$ mice. Subsequently, serum samples were obtained, and the concentration of IFN- $\gamma$ and IL- 4 was determined with ELISA kits (BD Bioscience) according to the manufacturer's instructions.

Statistics. Comparisons between the groups were made with the $t$-test for paired observations, and a $P$-value of $<0.05$ was considered significant.

SUPPLEMENTARY MATERIAL is linked to the online version of the paper at http://www.nature.com/mi

\section{ACKNOWLEDGMENTS}

We are grateful to Wilina Lim (Department of Health, The Government of Hong Kong) for providing us the A/Vietnam/1194/04 (H5N1) influenza virus strain, U. Suzuki and Dr Komase (Kitasato Institute, Saitama, Japan) for providing us the vaccines, and E. Berglund for reading and editing the manuscript. This work was partly supported by RIKEN Strategic Research Programs for R\&D (the President's Discretionary Fund), The Uehara Memorial Foundation, The Innovation Center in Kanagawa Academy of Science and Technology, Ministry of Health, Labor, and Welfare, and Research on Health Sciences focusing on Drug Innovation. T. Ichinohe is a research fellow of the Japan Society for the Promotion of Science.

\section{DISCLOSURE}

The authors declared no conflict of interest.

(C) 2008 Society for Mucosal Immunology

\section{REFERENCES}

1. Smidt, M.H., Stroink, H., Bruinenberg, J.F. \& Peeters, M. Encephalopathy associated with influenza A. Eur. J. Paediatr. Neurol. 8, 257-260 (2004).
2. Check, E. Bird flu: on border patrol. Nature 442, 348-350 (2006)

3. Renegar, K.B. Influenza virus infections and immunity: a review of human and animal models. Lab. Anim. Sci. 42, 222-232 (1992).

4. Jackson, L.A. et al. Safety of a trivalent live attenuated intranasal influenza vaccine, FluMist, administered in addition to parenteral trivalent inactivated influenza vaccine to seniors with chronic medical conditions. Vaccine 17, 1905-1909 (1999).

5. Piedra, P.A. et al. Safety of the trivalent, cold-adapted influenza vaccine in preschool-aged children. Pediatrics 110, 662-672 (2002).

6. Tamura, S., Tanimoto, T. \& Kurata, T. Mechanisms of broad crossprotection provided by influenza virus infection and their application to vaccines. Jpn. J. Infect. Dis. 58, 195-207 (2005).

7. Asahi, Y. et al. Protection against influenza virus infection in polymeric Ig receptor knockout mice immunized intranasally with adjuvant-combined vaccines. J. Immunol. 168, 2930-2938 (2002).

8. Nguyen, H.H. et al. Heterosubtypic immunity to influenza A virus infection requires $\mathrm{B}$ cells but not $\mathrm{CD} 8^{+}$cytotoxic T lymphocytes. J. Infect. Dis. 183, 368-376 (2001).

9. Takada, A. et al. Intranasal immunization with formalin-inactivated virus vaccine induces a broad spectrum of heterosubtypic immunity against influenza A virus infection in mice. Vaccine 21, 3212-3218 (2003).

10. Tumpey, T.M., Renshaw, M., Clements, J.D. \& Katz, J.M. Mucosal delivery of inactivated influenza vaccine induces B-cell-dependent heterosubtypic cross-protection against lethal influenza A H5N1 virus infection. J. Virol. 75, 5141-5150 (2001).

11. Asahi-Ozaki, Y. et al. Secretory IgA antibodies provide cross-protection against infection with different strains of influenza B virus. J. Med. Virol. 74, 328-335 (2004).

12. Hasegawa, H. et al. Protection against influenza virus infection by intranasal administration of hemagglutinin vaccine with chitin microparticles as an adjuvant. J. Med. Virol. 75, 130-136 (2005).

13. Tamura, S.I. \& Kurata, T. A proposal for safety standards for human use of cholera toxin (or Escherichia coli heat-labile enterotoxin) derivatives as an adjuvant of nasal inactivated influenza vaccine. Jpn. J. Infect. Dis. 53, 98-106 (2000).

14. van Ginkel, F.W., Jackson, R.J., Yuki, Y. \& McGhee, J.R. Cutting edge: the mucosal adjuvant cholera toxin redirects vaccine proteins into olfactory tissues. J. Immunol. 165, 4778-4782 (2000).

15. Mutsch, M. et al. Use of the inactivated intranasal influenza vaccine and the risk of Bell's palsy in Switzerland. N. Engl. J. Med. 350, 896-903 (2004).

16. Kronenberg, M. Toward an understanding of NKT cell biology: progress and paradoxes. Annu. Rev. Immunol. 23, 877-900 (2005).

17. Kawano, T. et al. CD1d-restricted and TCR-mediated activation of valpha14 NKT cells by glycosylceramides. Science 278, 1626-1629 (1997).

18. Taniguchi, M., Seino, K. \& Nakayama, T. The NKT cell system: bridging innate and acquired immunity. Nat. Immunol. 4, 1164-1165 (2003).

19. Ko, S.Y. et al. alpha-Galactosylceramide can act as a nasal vaccine adjuvant inducing protective immune responses against viral infection and tumor. J. Immunol. 175, 3309-3317 (2005).

20. Youn, H.J. et al. A single intranasal immunization with inactivated influenza virus and alpha-galactosylceramide induces long-term protective immunity without redirecting antigen to the central nervous system. Vaccine 25, 5189-5198 (2007).

21. O'Keeffe, J. et al. Diverse populations of T cells with NK cell receptors accumulate in the human intestine in health and in colorectal cancer. Eur. J. Immunol. 34, 2110-2119 (2004).

22. Harada, M. Down-regulation of invariant Valpha14 antigen receptor in NKT cells upon activation. Int. Immunol. 16, 241-247 (2004).

23. Jiang, X. et al. Cutting edge: critical role of CXCL16/CXCR6 in NKT cell trafficking in allograft tolerance. J. Immunol. 175, 2051-2055 (2005).

24. Campos, R.A. et al. Cutaneous immunization rapidly activates liver invariant Valpha14 NKT cells stimulating B-1 B cells to initiate T cell recruitment for elicitation of contact sensitivity. J. Exp. Med. 198, 1785-1796 (2003).

25. Galli, G. et al. CD1d-restricted help to B cells by human invariant natural killer T lymphocytes. J. Exp. Med. 197, 1051-1057 (2003).

26. Check, E. Avian flu special: is this our best shot? Nature 435, 404-406 (2005).

27. Benton, K.A. et al. Heterosubtypic immunity to influenza A virus in mice lacking IgA, all Ig, NKT cells, or $\gamma \delta$ T cells. J. Immunol. 166, 7437-7445 (2001).

28. Giaccone, G. et al. A phase I study of the natural killer T-cell ligand alphagalactosylceramide (KRN7000) in patients with solid tumors. Clin. Cancer Res. 8, 3702-3709 (2002). 


\section{ARTICLES}

29. Ichinohe, T. et al. Synthetic double-stranded RNA poly(l:C) combined with mucosal vaccine protects against influenza virus infection. J. Virol. 79, 2910-2919 (2005).

30. Nicolson, C., Major, D., Wood, J.M. \& Robertson, J.S. Generation of influenza vaccine viruses on Vero cells by reverse genetics: an $\mathrm{H} 5 \mathrm{~N} 1$ candidate vaccine strain produced under a quality system. Vaccine $\mathbf{2 3}$, 2943-2952 (2005).

31. Cui, J. et al. Requirement for Valpha14 NKT cells in IL-12-mediated rejection of tumors. Science 278, 1623-1626 (1997).

32. Shimaoka, T. et al. Critical role for CXC chemokine ligand 16 (SR-PSOX) in Th1 response mediated by NKT cells. J. Immunol. 179, 8172-8179 (2007)

33. Tamura, S. et al. Acceleration of influenza virus clearance by Th1 cells in the nasal site of mice immunized intranasally with adjuvant-combined recombinant nucleoprotein. J. Immunol. 156, 3892-3900 (1996).
34. Sever, J.L. Application of a microtechnique to viral serological investigations. J. Immunol. 88, 320-329 (1962).

35. Yetter, R.A., Lehrer, S., Ramphal, R. \& Small, P.A. Jr. Outcome of influenza infection: effect of site of initial infection and heterotypic immunity. Infect. Immun. 29, 654-662 (1980).

36. Tamura, S. et al. Antibody-forming cells in the nasal-associated lymphoid tissue during primary influenza virus infection. J. Gen. Virol. 79, 291-299 (1998).

37. Kuhn, R., Rajewsky, K. \& Muller, W. Generation and analysis of interleukin4 deficient mice. Science 254, 707-710 (1991).

38. Tagawa, Y., Sekikawa, K. \& Iwakura, Y. Suppression of concanavalin Ainduced hepatitis in IFN-gamma(-/-) mice, but not in TNF-alpha(-/-) mice: role for IFN-gamma in activating apoptosis of hepatocytes. J. Immunol. 159, 1418-1428 (1997). 